
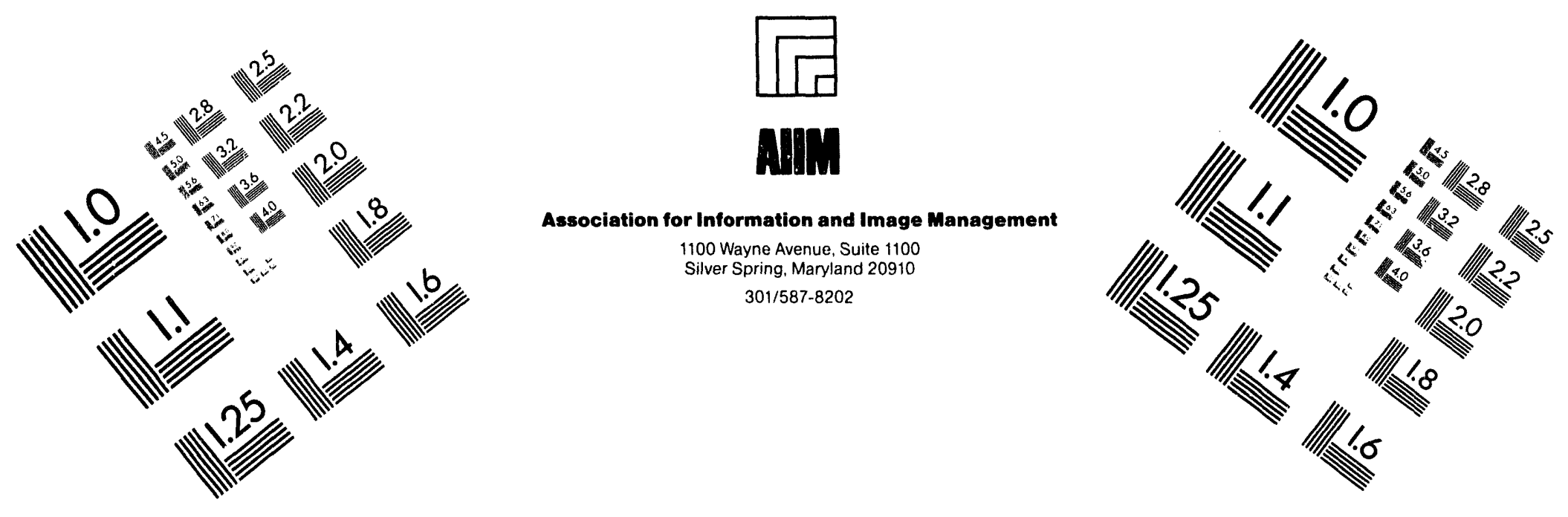

\title{
Centimeter
}

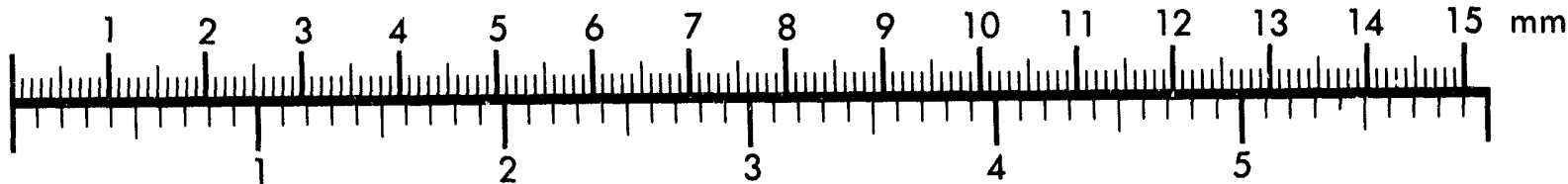

Inches
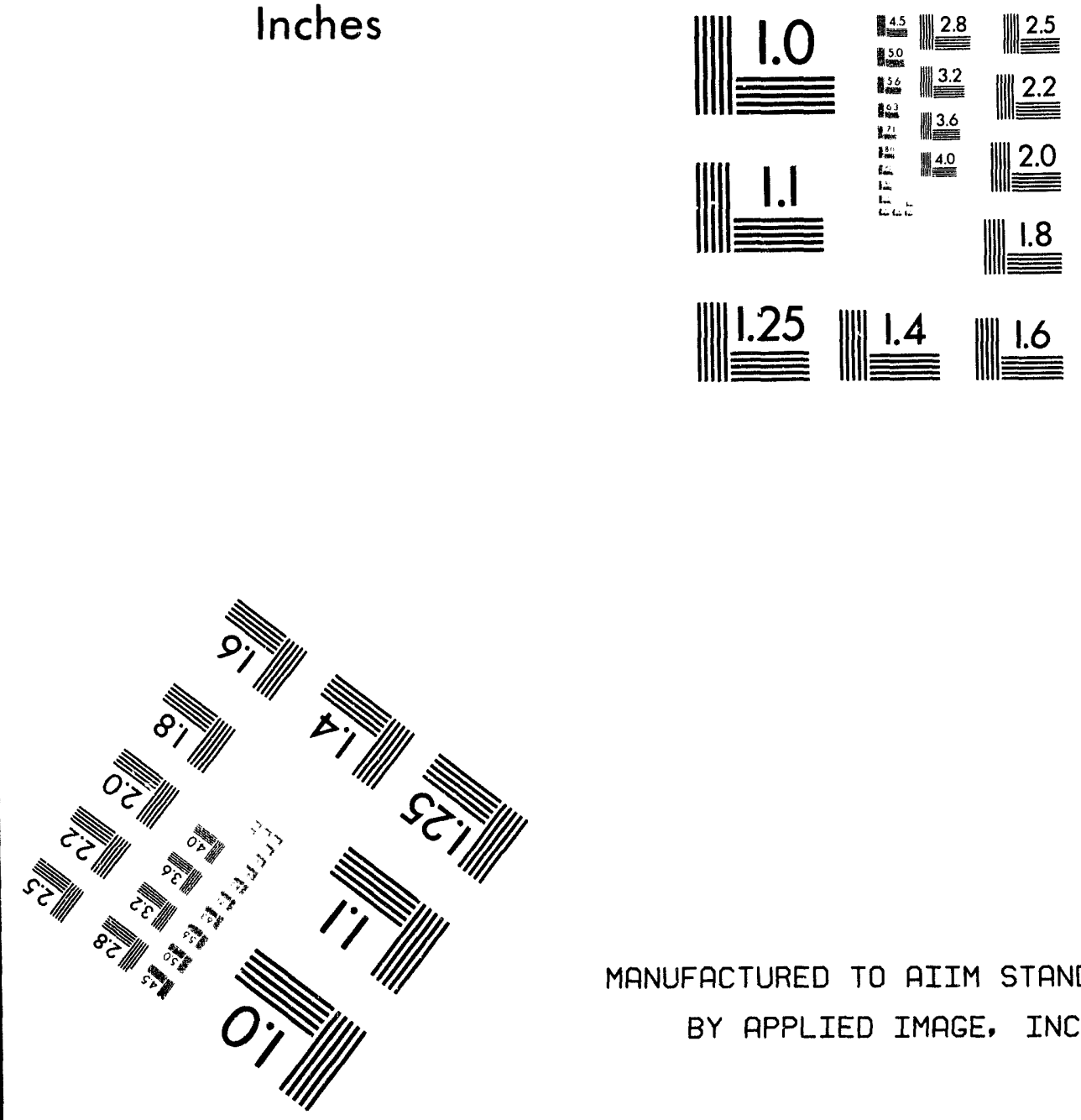

MANUFACTURED TO AIIM STANDARDS

BY APPLIED IMAGE, INC.

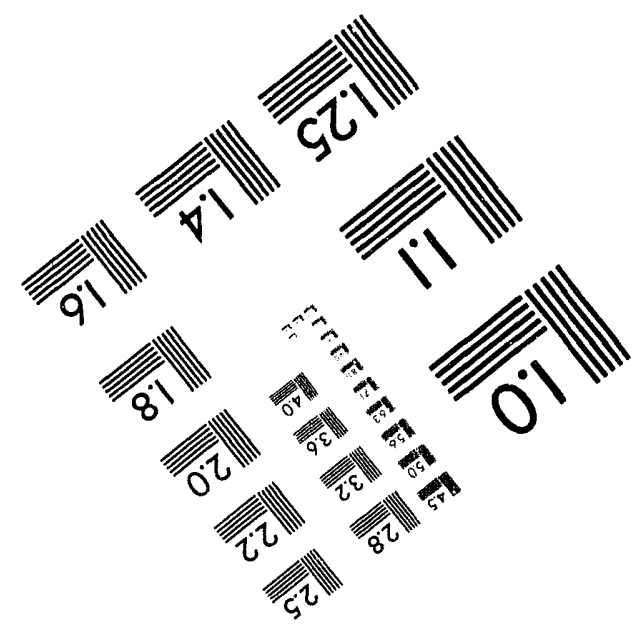



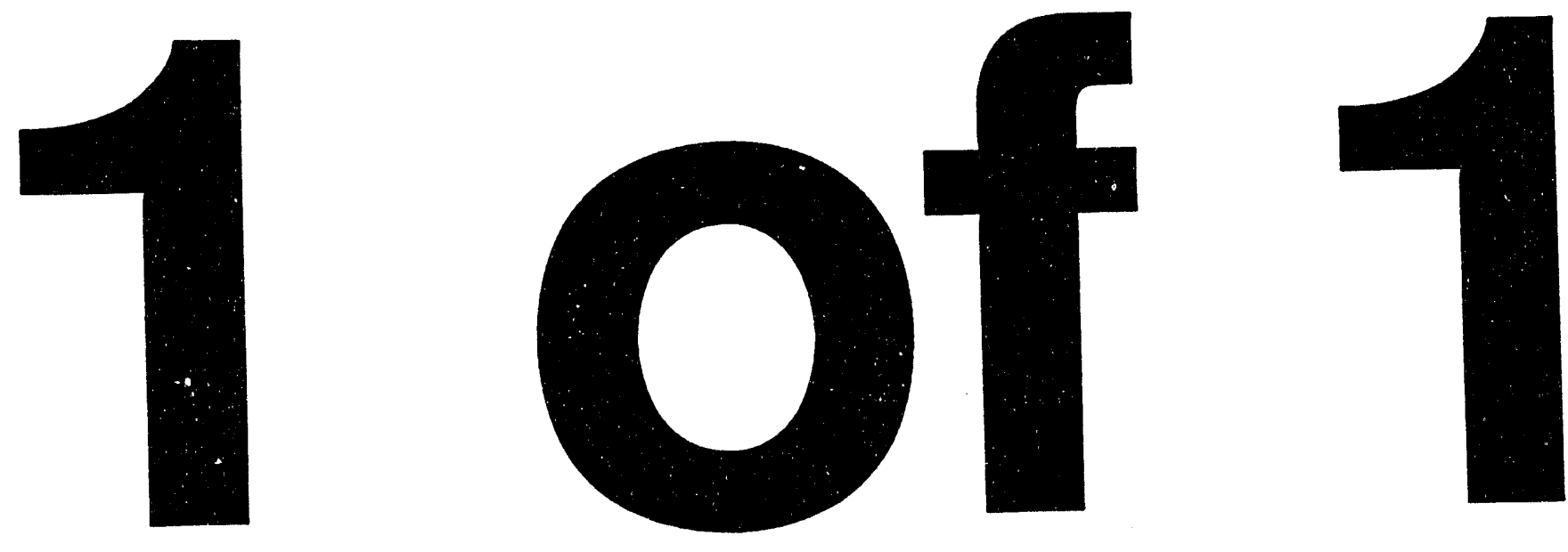


\title{
PROGRESS REPORT ON
}

\section{GRAIN BOUNDARIES}

R. W. Ballufĩi

P. D. Bristowe

Department of Materials Science and Engineering Massachusetts Institute of Technology, Cambridge, MA 02139

\author{
February 15, 1990 - October 15, 1990 \\ Massachusetts Institute of Technology \\ Cambridge, Massachusetts 02139 \\ Prepared for \\ U.S. Department of Energy \\ Under Contract DE-FG02-87ER45310
}

This report was prepared as an account of work sponsored by the United States Government. Neither the United States nor the United States Department of Energy, nor any of their employees, nor any of their contractors, subcontractors, or their employees, makes any warranty, express or implied, or assume any legal liability or responsibility for the accuracy, completeness, or usefulness of any information, apparatus, produce or process disclosed or represents that its use would not infringe privately owned rights. 


\section{CONTENTS}

1. INTRODUCTION

2. PERSONNEL

3. RESEARCH ACCOMPLISHED

3.1 Structural Studies

Dislocation structure of the $\mathrm{Ni} / \mathrm{Ag}$ heterophase boundary

Structural complexity in grain boundaries with covalent bonding

Relationship between microscopic properties of two semi-conducting grain boundaries and their orientations

Calculation of diffraction effects due to double positioning in (111) gold bicrystals

Sensitivity of diffraction profiles to grain boundary segregation

Solute segregation at grain boundaries in gold

Development of a 4-body interatomic potential for $S i$ and its use in defect calculations

3.2 Studies of Boundary Migration

A molecular dvnamics study of grain boundary migration without the participation of grain boundary dislocations

3.3 Study of Short-circuit Diffusion Along Grain Boundaries and Its Dependence on Boundary Structure

3.4 Development of Thin-film Deposition/Bonding Apparatus for the Manufacture of High Purity Bicrystals

4. LIST OF ALI REPORTS AND PUBLICATIONS ORIGINATING FROM. THE RESEARCH

REFERENCES 
1. INTRODUCTION

The present document is a progress report describing the work accomplished to date during the first year of our four-year grant (February 15, 1990 - February 14, 1994) to study grain boundaries under Grant DE-FG02-87ER45310. The research was focused on the following four major efforts:

(1) study of the atomic structure of grain boundaries by means of $x$-ray diffraction, transmission electron microscopy and computer modeling

(2) study of grain boundary migration

(3) study of short-circuit diffusion along grain boundaries

(4) development of Thin-Film. Deposition/Bonding Apparatus for the manufacture of high purity bicrystals.

Efforts (1), (2) and (3) were proposed initially for the period of the grant. Effort (4) developed out of an urgent need for an apparatus capable of producing high purity bicrystal specimens containing grain boundaries of controlled geometry. This apparatus will eventually assist us in essentially all aspects of our experimental work. Our accomplishments in each of these areas of research are described in more detail in the following. A list of all reports and publications originating from the research is presented in Section 4 . 
2. PERSONNEL

Prof. R. W. Ealluffi, Principal Investigator

Dr. P. D. Bristowe, Co-Principal Investigator

Dr. I. Majid, Research Associate

Dr. A. Amiri-Hezaveh, Postdoctoral Associate

Dr. Be-Jhen Jhan, Postdoctoral Associate (now at Paxametric Tech. Corp.)

Mr. C. Counterman, Graduate Student/Research Assistant (graduating date: 1991)

Mr. P. Dallot, Graduate Student/Research Assistant (graduating date: 1991)

Mr. Qing Ma, Graduate Student/Research Assistant (graduating date: 1992)

Mr. Dan Wang, Graduate Student/Research Assistant (graduating date: -1993)

Collaborative work was also performed by:

Prof. J. D. Joannopoulos (Dept. of Physics, MIT)

Dr. M. C. Payne (Cambridge University, U.K.)

Mr. T. Arias, Graduate Student/Research Assistant, Dept. of Physics,MIT

Mr. G. P. Francis (Cambridge University, U.K.)

Mr. E. Tarnow, Graduate Student/Research Assistant, Dept. of Physics, MIT 


\section{RESEARCH ACCOMPLISHED}

\section{$3: 1$ Structural studies}

\section{Dislocation structure of the $\mathrm{N} / \mathrm{Ag}$ heterophase boundary $[1]$}

The Ni/Ag interphase boundary structure in the parallel cube-on-cube orientation was studied for the boundary planes (001) and (011). Coarsely spaced dislocations were found in all annealed interfaces; the origin of these was apparently differential thermal expansion of the bicrystal film. A detailed search for intrinsic periodic structure consistent with the 0-lattice construction was made using $\mathrm{X}$-ray diffaction, electron diffraction and transmission electron microscopy. No evidence for this could be found; such a relaxation, if present, is very weak. It is unlikely that such a small relaxation could account for the energy minimum associated with the parallel cube-on-cube orientation found by previous investigators.

\section{Structural complexity in grain boundaries with covalent bonding 121}

The structural properties of two short-period twist boundaries in germanium were explored using a state-of-the-art total energy calculation. The structures of these boundaries were found to be very complex, with boundary bonds that are distorted and weak. These systems were found to exhibit a large degeneracy in the number of local energy minima. Thus the boundaries have difficulty in arriving at a locally ordered state. The situation may be unique to the semiconducting twist grain boundaries due to the inherent frustration present between the tendency to form directional bonds and the imposed twist geometry which makes the bond formation improbable. This study focused on the energy, coordination, volume change and 
electronic states characteristic of the local minima. A trend towards dimerization was found especially in the highest angle twist boundary.

Relationship between microscopic properties of two semiconducting grain boundaries and their orientations [3]

The microscopic structure of a tilt and a twist boundary in germanium was explored using a state-of-the-art total energy calculation. The structure of the tilt boundary $(\Sigma-5(310))$ was found to be simple as it exhibits a well-defined minimum energy structure, consistent with previous experinental and theoretical results on this and other tilt boundaries. The structure of the twist boundary $(\Sigma=5(100))$, however, was found to be very complex. The boundary bonds are distorted and weak, and their weakness makes the twist boundary exhibit a wealth of local energy minima. The different types of energy minima for the twist boundaries were identified and studied in some detail. The consequence of the orientation of the two crystal grains upon the microscopic structure of the boundaries was summarized and comments were made concerning future applications of these results.

\section{Calculation of diffraction effects due to double positioning in}

\section{(111) gold bicrystals 141}

The diffraction effects from double positioned [111] twist boundaries were calculated using an embedded atom model for gold. It was found that the kinematical scattering from any two boundaries related by double positioning is always superimposed. However, in a limited misorientation range near $30^{\circ}$, the superimposed intensities in the boundary plane are equal and can therefore 
be separated. Consequently, the projected atomic structure of each doubly positioned boundary can be determined by $x$-ray diffraction. It was found that the displacement field of [111] twist boundaries is generally characterized by rotational-like displacements centered around ' $O$ ' lattice elements. Since these displacements are largest for near $-\Sigma 1$ and near $-\Sigma 3$ orientations, these boundaries have the largest scattering power per unit area.

Sensitivity of diffraction profiles to grain boundary segregation $[5]$ In this study, the relrod profiles of a number of weak grain boundary reflections were computed with and without the presence of substitutional impurities. The $\Sigma 13\left(22.6^{\circ}\right)$ [001] twist in gold was chosen since, for this system, a number of relrod profiles have been measured that exhibit fine structure [6]. To perform the calculations, a molecular statics method was used together with embedded-atom potentials [7]. The first objective was to compare the measured and computed relrod profiles for pure gold to establish that the fine structure is intrinsic to the grain boundary. The second objective was to determine by calculation the sensitivity of these relrods to the presence of low concentrations of impurity, in this case nickel. It was found that many of the relrods respond in such a way as to make them useful indicators of grain boundary segregation. 


\section{Solute segregation at grain boundaries in gold}

Monte Carlo calculations are in progress to determine the effect of silver and nickel segregation on the structure of a number of grain boundaries in gold. The aim is to compare the results with diffraction experiments (see below) being performed in parallel on similar systems. Three principal parameters are being controlled to determine their effect on structure and structure factor. These are the boundary misorientation, the solute concentration and the temperature. Preliminary results indicate that for the Ag-Au system, even $50 / 50$ alloys yield little grain boundary segregation or ordering effects and consequently the relative boundary structure factars are very similar to that of pure gold boundaries. On the other hand, small concentrations of nickel in gold boundaries are predicted to have diffraction effects which should be measurable.

In the experimental part of this work, thin-film bicrystal specimens of Ag-Au and Ni-Au alloys containing controlled boundaries of the desired geometry have been prepared and have been heat-treated to produce equilibrium solute segregation at the grain boundaries. X-ray diffraction experiments are in progress to determine grain boundary structure factors which will be compared with those calculated as described above. These studies will be the first experiments capable of obtaining information about the detailed arrangement of solute atoms in segregated grain boundaries. 
Development of a 4 -body interatomic potential for Si and its use in defect calculations

A new empirical formalism has been applied to describe covalent bonding in silicon. The study is motivated by a need to simulate defect structures and microscopic processes in covalent materials where the atomic coordinations are far from ideal and where many-body effects are important. A prototype potential has been constructed based on the following approach. The total energy is expanded into 4-body terms in which each term except the last is exact. Thus, the last term is an effective screening potential. A new and general set of internal coordinates derived from symmetry considerations is used to define the 4-body potential. A functional form for the potential is constructed and the adjustable parameters are fitted to ab-initio pseudo-potential calculations of structures with 2,3 and 4 silicon atoms. At present, the new potential is being tested with respect to the known bulk properties of silicon. Once a satisfactory potential is found, it will be applied to a number of grain boundary siructures of interest.

\subsection{Studies of Boundary Migration}

A molecular dynamics study of grain boundary migration without the participation of grain boundary dislocations $[8,9]$

In this study, we were concerned with determining a possible atomistic mechanism for the migration of high-angle grain boundaries that does not involve grain boundary dislocations or other defects. The study was motivated 
by our previous experimental observations [10] that grain boundary

dislocations do not appear to be involved with grain boundary migration. To study the details of such a mechanism, a dynamic computer simulation was performed on a model grain boundary system which does not contain or generate dislocations but is designed to encourage migration. The chosen grain boundary model consisted of a stack of pure steps (or ledges) embedded in a short-period high-angle grain boundary and arranged so as to form a pyramid-like structure. The grain boundary plane was therefore effectively curved and had an overall hemispherical shape. Capillary forces therefore exist which should induce the boundary to move in a direction which minimizes its surface area in a manner analogous to that observed in the experiments of Babcock and Balluffi [10]. This is the first time a simulation study has been made on grain boundary migration in which the boundary is initially curved. Previous molecular dynamics calculations have been performed on flat grain boundary structures in which the model was either relatively small allowing the entire boundary to slide as well as migrate or migrate locally producing roughness effects due solely to thermal fluctuations. Our results indicated that the qualitative shuffle mechanism proposed by Babcock and Balluffi [10] to explain their observations of migrating bourdaries in the absence of SGBDs is appropriate, at least in the model systems studied. In particular, it was shown that the boundaries moved by local conservative shuffles of atoms or groups of atoms such that one adjoining crystal grew at the experise of the other. The shuffles often took the form of correlated rotational displacements about an axis normal to the boundary and involved a characteristic group of four atoms. 
3.3 Study of Short-circuit Diffusion Along Boundaries and Its Dependence on Bourdary Structure

Grain boundary chemical diffusivities for a series of (001) tilt boundaries in the $\mathrm{Au} / \mathrm{Ag}$ system were measured by the surface accumulation technique. CSL boundaries of low $\Sigma($ i.e., $5,13,17,25$ ) and also more ordinary boundaries with tilt angles around those special orientations were selected. Novel techniques were developed to make the diffusion specimens, which consisted of a silver layer deposited on a gold layer (each layer - $1000 \AA$ thick) and which contained an array of parallel grain boundaries of the same kind running perpendicular to the surfaces. At temperatures between $100^{\circ} \mathrm{C}$ and $250^{\circ} \mathrm{C}$, lattice diffusion was frozen out, and the $\mathrm{Ag}$ and $\mathrm{Au}$ atoms diffused from their original surfaces, through the grain boundaries, to the opposite surfaces where they accumulated and were measured by Auger spectroscopy. The grain boundary diffusion parameters were derived from the surface accumulation rate data by using computer modeling techniques. Special efforts were made to separate structural effects from other influential effects, sich as segregation and diffusion-induced grain boundary migration. The dependence of the grain boundary diffusion on the boundary structure, as well as the applicabilities of existing structural models in describing boundary diffusion effects, were considered. 


\subsection{Development of Thin-film Deposition/Bonding Apparatus for the}

Manufacture of High Purity Bicrystals

For a number of years, a major aspect of our work has been the use of special specimens prepared by vapor depositing epitaxial single crystal thin films and then welding them together face-to-face at predetermined misorientations to produce thin-film bicrystal specimens. This procedure produces specimens containing grain boundaries in their midplanes which are thin enough to allow detailed studies of the boundary structure by means of transmission electron microscopy and also x-ray diffraction. Up until now, we have concentrated on studying $A u$ and/or Ag specimens which have been produced by welding $\mathrm{Au}$ and/or Ag films together (after deposition in vacuum) in air. We have been concerned that impurities may have been introduced by the exposure to air. Furthermore, our studies have been confined to $\mathrm{Au}$ and $\mathrm{Ag}$, since the surfaces of these metals can be briefly exposed. to air during the welding without pisking up excessive contamination.

In view of the above problems and limitations, we have embarked on the construction of a UHV thin-film deposition and bonding apparatus which will allow us to produce thin-films of a wide variety of metals and weld them together in situ in a single apparatus under UHV conditions without any exposure to air. The resulting specimens should be of high perfection and free of impurities, and, hence, should be ideal for further basic studies of the structure and properties of grain boundaries (and also heterophase boundaries) in a wide range of materials, including metals and semi-conductors. 
Figure 1 gives schematic drawings of the side and top views of the nverall apparatus as planned.

a) Fast entry load-lock system:

The load-lock system allows quick and easy loading and unloading of substrates and evaporated films in and out of the deposition chamber without breaking the UHV system. The substrate crystals are first mounted onto two holders, each having 6 to 12 sites. In order to obtain bicrystals of specified misorientations in the subsequent welding operation, one of the substrate holders has the ability to produce variable but precise rotational positioning. The substrates are then loaded onto the internal transporter through the quick access door of the specimen transfer load-lock chamber. After the specimen transfer chamber is pumped to $10^{-9}$ Torr by a $80 \mathrm{l} / \mathrm{s}$ turbo molecular pump, backed by a mechanical pump, the C-lock gate valve (1) is opened for access into the deposition chamber. The substrates are then transferred to the deposition chamber by a 18 in magnetically coupled rotary-linear transporter rod.

b) Deposition system:

The deposition system consists of a 21 in $\times 18$ in UHV chamber with a liquid nitrogen shroud assembly, a UHV precision specimen manipulator, a 10 $\mathrm{KeV}$ Reflection High Energy Electron Diffraction (RHEED) gun, a RHEED phosphorus screen, a $2 \mathrm{KeV}$ ion sputtering gun and deposition assembly. The chamber is pumped by a $400 \mathrm{l} / \mathrm{s}$ ion and a titanium sublimation pump with a liquid nitrogen cryo-shield. 
The UHV precision specimen manipulator, with $\mathrm{x}, \mathrm{y}, \mathrm{z}$ rotary $\left(360^{\circ}\right)$ and co-axial motions, is mounted on top of the deposition chamber in order to supiport the specimen holders. Because of the maneuvering abilities of the manipulator, the substrates can be placed in front of the RHEED system, deposition assembly or a sputtering ion gun. Eefore the thin-film deposition, the substrate surfaces are sputter-cleaned by a $2 \mathrm{KeV}$ ion gun. The deposition system consists of four $20 \mathrm{cc}$ effusion cells which are mounted onto four 4.5 in $\times 6$ in water-cooled ports, with a multiple shutter mechanism fo: beam selection. The whole assembly is covered by an extra liquid nitrogen shroud and then mounted at the bottom of the chamber by a 24 in flange.

During deposition, the growth modes and crystal structure of the thin films are monitored by RHEED. The layer thickness is controlled by observing the RHEED intensity variation (Phase Locked Epitaxy), which is a function of the atomic layer thickness during the growth.

c) The UHV bonding system:

The bonding system consists of a 10 in $\times 18$ in water-cooled UHV chamber, an interlock specimen transfer tube and two linear motion feedthroughs for the mounting of two specimen holders. The extra ports in the chamber are for potential Auger electron spectroscopy or Auger electron microscopy and a scanning ion sputtering gun. 
The chamber is pumped through the deposition chamber with $400 \mathrm{l} / \mathrm{s}$ ior, cryo and TS pumps. In order to increase the pumping speed during the bonding operation, an extra $400 \mathrm{l} / \mathrm{s}$ ion pump is attached directly to the chamber.

After the deposition of the epitaxial thin films, the specimen holders are transferred to the UHV bonding chamber by a 36 in long, magnetically coupled rotary-linear transport rod. The specimen holders are loaded onto two linear motion feedthroughs for bonding by hot-pressing. The hot-pressing is accomplished by forcing the thin-film crystals together face-to-face by means of rods running through the linear motion feedthrough ports. During this operation, the specimen holders (and the specimens themselves) are heated resistively to the desired welding temperature.

At the present time, the main body of the apparatus has been assembled and the various manifolds are being. vacuum tested. All of the auxiliary equipment is being installed and tested, and it is anticipated that the first deposition and bonding runs will be started within the next few months. 
4. LIST OF ALI REPORTS AND PUBLICATIONS ORIGINATING FROM THE RESEARCH

1) T. A. Bamford, "An Investigation of the Dislocation Structure of the Ni/Ag Phase Boundary", Mat.Res.Soc.Proc., accepted for publication: DOE/ER/45310-20.

2) E. Tarnow, P. Dallot, P. D. Bristowe, J. D. Joannopoulos, G. P. Francis and M. C. Payne, "structural complexity in grain boundaries with covalent bonding", Phys. Rev, B, 42, 3644 (1990): DOE/ER/45310-21.

3) Re-Jhen Jhan and P. D. Bristowe, "Dynamical simulation of the motion of curved grain boundaries composed of pyramidal-shaped ledges", Mat. Res. Soc. Proc. 193, 189 (1990): DOE/ER/45310-22.

4) E. Tarnow, T. Arias, P. D. Bristowe, P. Dallot, G. P. Francis, J. D. Joannopoulos and M. C. Payne, "The relationship between the microscopic properties of two semiconducting grain boundaries and their orientation", Mat. Res. Soc. Proc. 193, 235 (1990): DOE/ER/45310-23.

5) D. Wang and P. D. Bristowe, "Calculation of diffraction effects due to double positioning in (111) gold bicrystals", Phys.Stat.Sol.(b), 161 (1990): DOE/ER/45310-24.

6) Re-Jhen Jhan and P. D. Bristowe; "A molecular dynamics study of grain boundary migration without the participation of secondary grain boundary dislocations", Scripta Metall., 24, 1313 (1990): DOE/ER/45310-25.

7) P. D. Bristowe, "The sensitivity of diffraction profiles to grain boundary segregation", Scripta Metall., accepted for publication: DOE/ER/45310-26.

8) R. W. Balluffi and P. D. Bristowe, "Progress Report on Grain Boundaries", DOE/ER/45310-27. 


\section{REFERENCES}

1) T. A. Bamford, "An Investigation of the Dislocation Structure of the Ni/Ag Phase Boundary", Mat.Res.Soc.Proc., accepted for publication: DOE/ER/45310-20.

2) E. Tarnow, P. Dallot, P. D. Bristuwe, J. D. Joannopoulos, G. P. Francis and M. C. Payne, "Struct" cal complexity in grain boundaries with covalent bonding", Phys. Rev. B, 42, 3644 (1990): DOE/ER/45310-21.

3) E. Tarnow, T. Arias, P. D. Bristowe, P. Dallot, G. P. Francis, J. D. Joannopoulos and M. C. Payne, "The relationship between the microscopic properties of two semiconducting grain boundaries and their orientation", Mat. Res. Soc. Proc. 193, 235 (1990): DOE/ER/45310-23.

4) D. Wang and P. D. Bristowe, "Calculation of diffraction effects due to double positioning in (111) gold bicrystals", Phys.Stat.Sol.(b), 161 (1990): DOE/ER/45310-24.

5) P. D. Bristowe, "The sensitivity of diffraction profiles to grain boundary segregation", Scripta Metall., accepted for publication: DOE/ER/45310-26.

6) M. R. Fitzsimmons and S. L. Sass, Acta Metall. 37, 1009 ((1989).

7) S. M. Foiles, M. I. Baskes and. M. S. Daw, Phys.Rev. B 33 , $7983(1989)$

8) Re-Jhen Jhan and P. D. Bristowe, "Dynamical simulation of the motion of curved grain boundaries composed of pyramidal-shaped ledges", Mat. Res. Soc. Proc. 193, 189 (1990): DOE/ER/45310-22.

9) Re-Jhen Jhan and P. D. Bristowe, "A molecular dynamics study of grain boundary migration without the participation of secondary grain boundary dislocations", Scripta Metall., 24, 1313 (1990): DOE/ER/45310-25.

10) S. E. Babcock and R. W. Balluffi, Acta Metall. 37, 2367 (1989). 

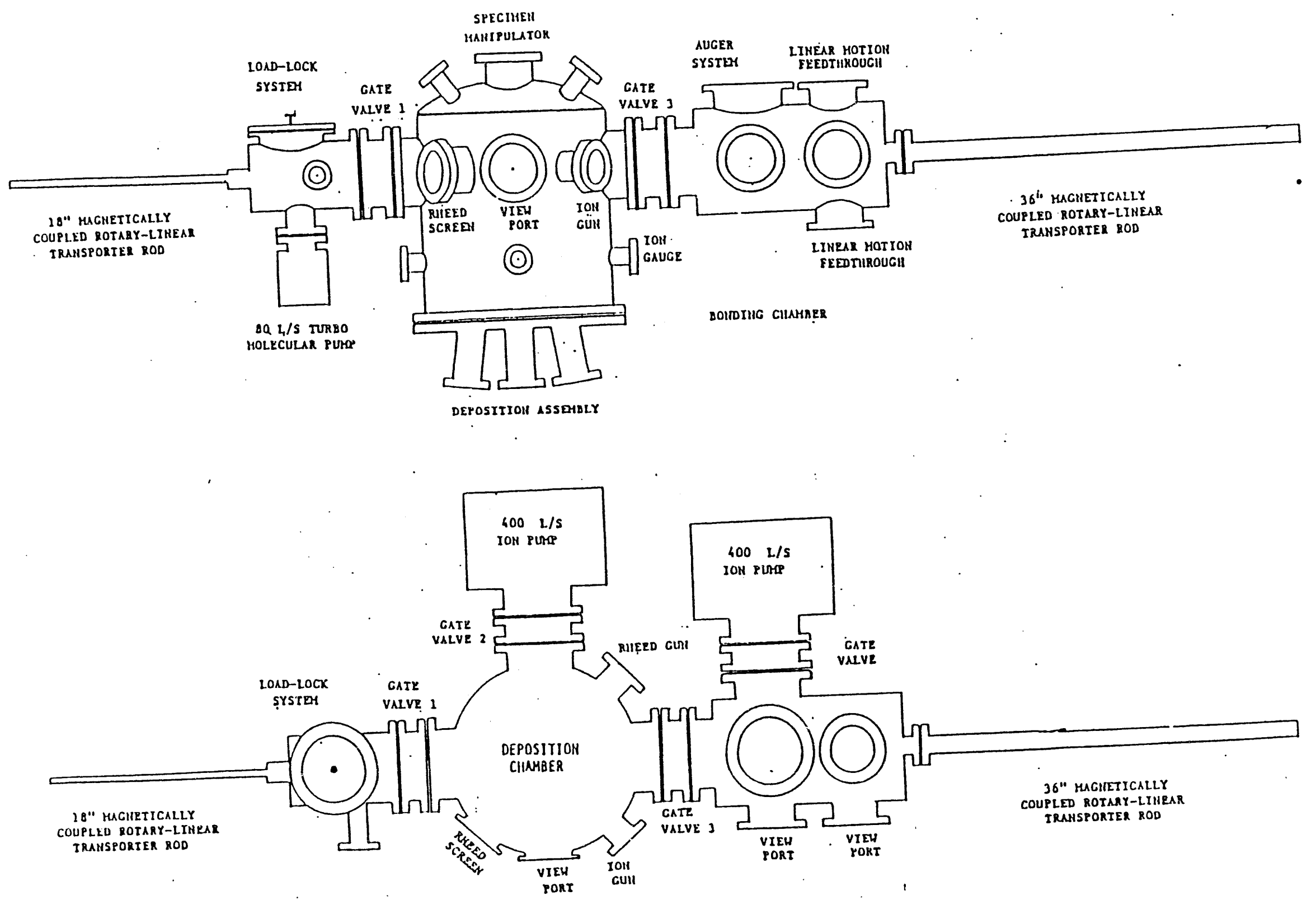

Figure 1 

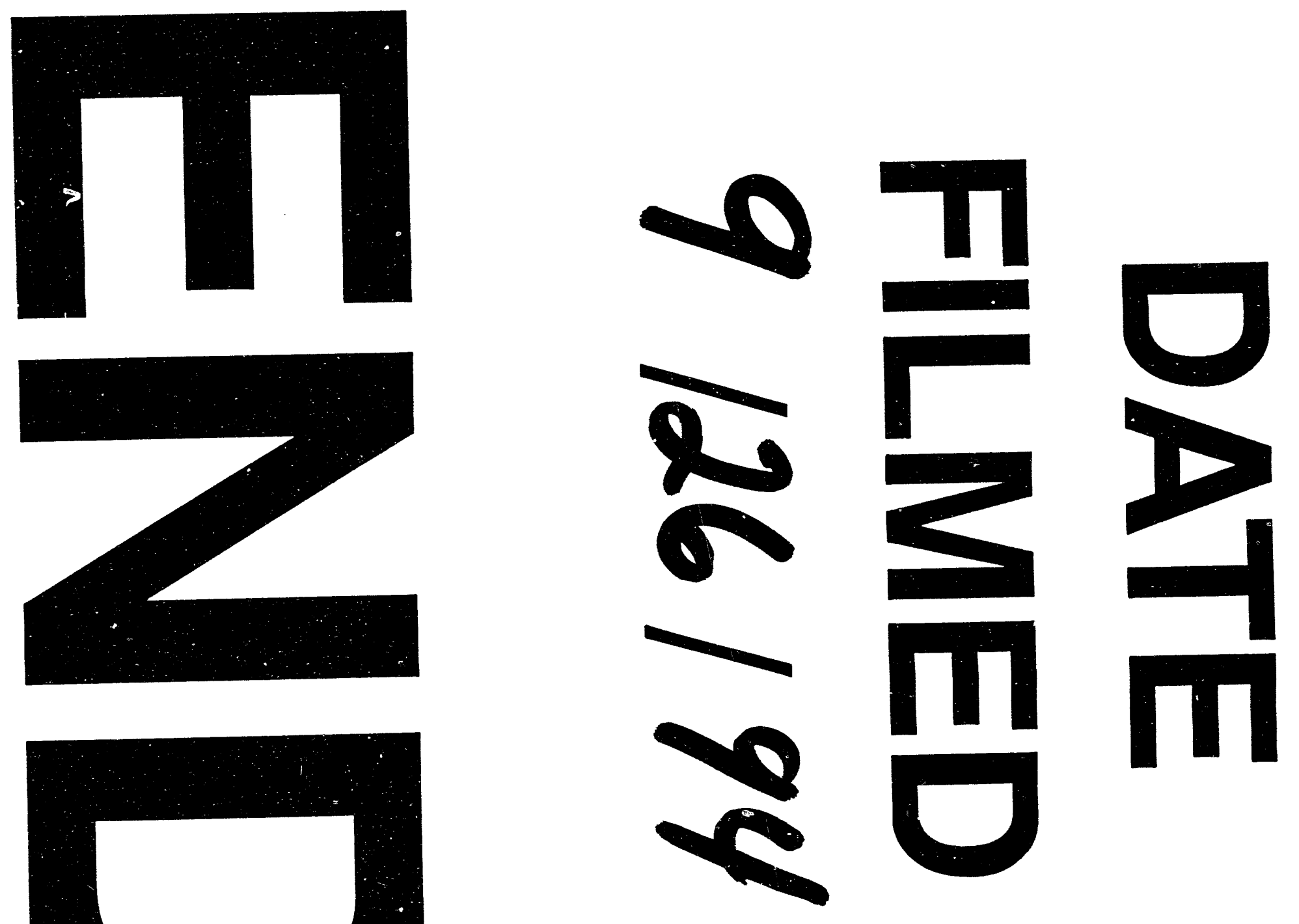
